“C 2016 IEEE. Personal use of this material is permitted. Permission from IEEE must be obtained for all other uses, in any current or future media, including reprinting/republishing this material for advertising or promotional purposes, creating new collective works, for resale or redistribution to servers or lists, or reuse of any copyrighted component of this work in other works." 


\title{
Multi-relational Social Recommendations via Multigraph Ranking
}

\author{
Mingsong Mao, Jie Lu, Senior Member, IEEE, Guangquan Zhang, Member, IEEE and Jinlong Zhang
}

\begin{abstract}
Recommender systems aim to identify relevant items for particular users in large-scale online applications. The historical rating data of users is a valuable input resource for many recommendation models such as collaborative filtering (CF), but these models are known to suffer from the rating sparsity problem when the users or items under consideration have insufficient rating records. With the continued growth of online social networks, the increased user-to-user relationships are reported to be helpful and can alleviate the $\mathrm{CF}$ rating sparsity problem. Although researchers have developed a range of social network-based recommender systems, there is no unified model to handle multi-relational social networks. To address this challenge, this paper represents different user relationships in a multigraph and develops a multigraph ranking model to identify and recommend the nearest neighbours of particular users in high-order environments. We conduct empirical experiments on two real-world datasets, Epinions and Last.fm, and the comprehensive comparison with other approaches demonstrates that our model improves recommendation performance in terms of both recommendation coverage and accuracy, especially when the rating data are sparse.
\end{abstract}

Index Terms-Recommender Systems, Graph Ranking, Random Walks, Social Network Analysis

\section{INTRODUCTION}

$\mathrm{T}$ HE explosive growth of web technologies in recent years, as well as the volume of Internet users, promotes the advent of large-scale online applications. The recommender system is a type of automation response framework for a requester (the user) to obtain personalized suggestions for resources (the items). Traditionally, users' explicit ratings on items are utilized as the major input resource for recommendation models such as Collaborative Filtering (CF), in which the main concern is the problem of sparse rating data. With the dramatic growth of social networking tools, user-to-user relationships are emerging as another source of information for amalgamating peoples' opinions which can be incorporated to improve pure, rating-based CF. It has been reported that various

Mingsong Mao, Guanquan Zhang (guangquan.zhang@uts.edu.au), and Jie Lu (jie.lu@uts.edu.au) are with the Centre for Quantum Computation and Intelligent Systems, Faculty of Engineering and IT, University of Technology Sydney, Sydney, Australia.

Mingsong Mao and Jinlong Zhang (jlzhang@mail.hust.edu.cn) are with the Institute of Management Information, School of Management, Huazhong University of Science and Technology, Wuhan, China.

Mingsong Mao (mingsongmao@jxufe.edu.cn) is with the School of Information Technology, Jiangxi University of Finance and Economics, Nanchang, China. types of user relationships have been studied in this field, including both explicit social relationships [1]-[6] and implicit correlations of users [7]-[15]. Despite the success of each independent model, a new challenge is that people are often connected by multiple types of relationships simultaneously, but few studies have sought to handle such multi-relational environments for recommendation [16]. Motivated by the need to attempt this, this paper proposes a multigraph ranking-based recommendation model that is able to identify the overall nearest neighbour users for a particular user from complex social relations and then find his/her potentially interested items.

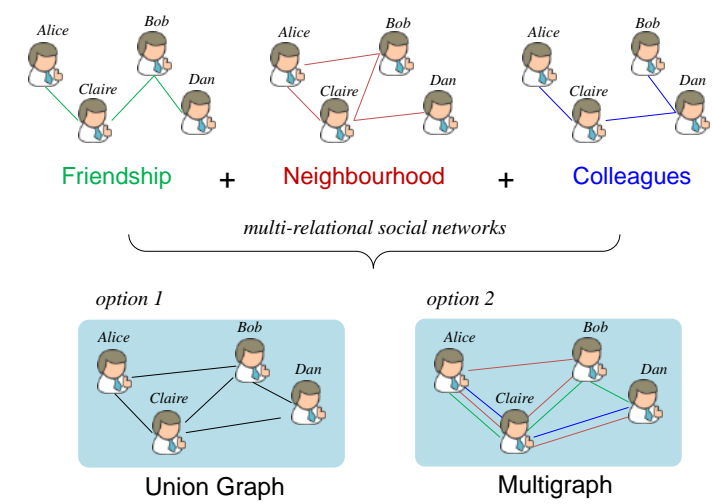

Fig.1 Modelling multi-relational social networks using a union graph vs. a multigraph

A toy example of multi-relational social networks is shown in Fig.1, where four users are surrounded by three types of relationships: friendship, neighbourhood and colleague relationships. A simple method to handle these high-order relationships is to aggregate the different relationships between each two users into a single relationship (option 1 in Fig.1), and finally to build a union simple graph connecting all users. As a result, however, the structural information of the original relationships will be lost [17] in this way. The other option is to build a multigraph model (option 2 in Fig.1) to retain all the structural information. Unlike conventional graphs, a multigraph is a special type of graph which is permitted to have multiple edges, i.e., edges that have the same end nodes. The three types of social relationships shown in Fig.1 can be naturally represented as a multigraph. In this paper, we propose a multigraph ranking model that is able to identify nearest neighbour users in multi-relational social networks, and can produce recommendations in the same way as traditional $\mathrm{CF}$ approaches. This work will have two main components and corresponding innovations, as follows. First, it proposes a 
random walk model for single social network propagation to enrich original social data. Second, it proposes a multigraph ranking model to identify the overall closeness between users in multi-relational social networks. The social network propagation model is employed as a preliminary process to enrich the original social data, and the multigraph ranking model undertakes the next step to identify the nearest neighbour users for the purpose of making recommendations.

The rest of this paper is organised as follows. In Section II, we review existing social network-based recommender systems that incorporate explicit or implicit relationships of users to improve pure CF approaches. In Section III, we propose a random walk model for social network propagation. Section IV introduces the generation of a multigraph model from different user-to-user relationships that may appear in a recommender system. Next, a multigraph ranking model is developed in Section V, where the regularization framework of simple graph ranking is imported and improved. In Section VI, empirical experiments are conducted with two real-world datasets from the online applications of Epinions and Last.fm. It demonstrates the superiority of our approach in terms of both recommendation coverage and accuracy. We summarize our findings and future study in the last section.

\section{RELATED WORK}

\section{A. Recommender Systems}

The task of a recommender system is to help users to identify items of personal interest from a large number of choices in an online application. An intuitive way to achieve this is to seek items that have similar attributes to those items that have been preferred by a user previously; this information is usually stored as a content-based user "profile", which becomes the key component of content-based (CB) approaches [18]. In contrast, collaborative filtering (CF) approaches [19] do not rely on item content descriptions but draw user profiles directly from users' numeric ratings to items, e.g., on scale of 1 to 5. A particular user is profiled by his/her rating records, storing which items were liked or disliked. By comparing this user's ratings to other users' ratings on common items, the users who have similar preferences can be identified as neighbour users. With the neighbourhood of a given user established, the possible rating of an unknown item can be aggregated from the known ratings of other neighbour users if they have already rated this item. It is also possible to implement $\mathrm{CF}$ from item perspective, i.e., by generating predictions based on the ratings of similar items that acquired similar ratings from different users [20]. Although various CF models have been applied in many applications and have achieved success, a major limitation is the rating sparsity problem, which means that it is difficult or impossible for predictions to be generated in pure rating-based CF models if the related users or items have insufficient historical ratings.

\section{B. User Relationships in Recommender Systems}

Our review shows that a social network between recommender system users can be established from three types of resources: 1) preference correlations derived from ratings, 2) explicit social relationships provided by systems, or 3) implicit correlations derived from user-contributed information.

\section{1) User Preference Correlations}

As is known, quantified preferences such as user ratings have been widely utilized to construct the preference correlations of users, such as Pearson Correlation and Cosine similarity measurement, in conventional $\mathrm{CF}$ approaches. Binary experience information has also been exploited for recommender systems. For example, in the ItemRank model proposed by Gori and Pucci [21], a correlation network of items is built using the binary user-rating-item information. This study only considers item correlations, but we believe that a correlation network of users can also be established based on such binary information. Some studies suggest that implicit trust relationships can be generated from users' rating behaviours [9], [12], [14], [22], [23]. Commonly, these models assume that users will trust others who are found to hold similar opinions consistently. For example, O'Donovan et al. use rating-derived implicit trust instead of rating similarities between users to conduct collaborative filtering and obtain good outcomes [2]. Yuan et al. develop an implicit trust metric between users based on their rating similarities [12]. As a result, the implicit trust network is denser than the original rating similarities, so the sparsity problem is well eliminated. In [23], Shambour et al. also consider the transitivity of implicit trust. For users that are not directly trusted, the indirect trustworthiness is inferred using a trust propagation model. The enriched implicit trust network is then integrated with rating similarities to develop a so-called trust-enhanced approach for recommendations. In summary, various types of preference correlations can be derived from rating behaviours in addition to rating similarities.

\section{2) Explicit Social Relations}

Increasingly, online systems involve social networking tools to enable customers to interact with each other directly, in the form of online friendships, interest groups, etc. In academia, researchers have contributed to the incorporation of social networks to improve recommendations, especially in the case of sparse rating data [1]-[6]. In life, people often resort to their friends for suggestions. It is therefore tempting to incorporate users' trust relationships in social networks to enhance collaborative filtering. A few systems provide weighted trustworthiness between users so that the trust scores can be directly utilized as the weights to find neighbour users. For example, in the FilmTrust recommender system proposed by Golbeck [24], users are required to provide a trust rating in the range of $[0,1]$ when a new person is added to the trust list. However, the majority of social network-enabled systems only provide binary relations between users such as "who trusts who" or "who is linked to whom". Graph searching techniques are often employed for social network propagation, in which some parameters need to be tuned to control the search breadth or depth [2], [3], [23]. Take the TidalTrust model in [2] for instance. It is a modified Breadth-First-Search (BFS) model that polls indirect trustworthiness from directly trusted persons. A similar model called MoleTrust is developed in [3] by Massa et al. Deeper-level trust inference models have also been 
proposed, as in [23]. For instance, if no one-level trustees know the target user, the polling process is recursively conducted at deeper levels. In addition, the maximum allowed searching depth is controlled by new parameters.

3) Implicit User Correlations

Implicit correlation networks of users can be derived from broader user-contributed information such as behaviour history and feedback data. People are usually thought to be related if they share common characteristics or have engaged in similar behaviours. In the website bookmarks recommender systems of [8], Shiratsuchi et al. construct a type of "co-citation" relationship network of users as the basis for exploring the implicit correlations of users to see whether they have similar interests surfing the Internet. Lopes et al. [11] develop an academic collaboration recommender system to help scientists to find potential opportunities for cooperation. An implicit social network of scientists can be generated based on their "co-authoring" relationships. Social tags have also been imported to construct correlations of users. For example, Liang et al. [10] map user tags into the item taxonomy space as the representations of users, and users are thus able to initiate tag-based correlations.

In summary, more diverse correlations of users can be extracted as new resource aspects for conducting or enhancing collaborative filtering, particularly for systems with sparse rating data. There are many related studies that exploit explicit or implicit correlations of users in specific domains, but it lacks general models that can handle multi-relational correlations derived from diverse information, which is the aim of this study.

\section{Fusion Methodology of Social Networks}

Incorporating social networks with conventional $\mathrm{CF}$ approaches has gained much attention in the literature, and can be categorized in three ways:

Post hoc combination. This refers that each input resource is investigated in separate recommendation approaches, the results of which are later combined [23], [25], [26].

Unified models. Unified models are usually applied for systems with complex networks of users, items and/or contextual information. Examples include the multi-partite graph models [5], hypergraph models [27] and cross domain multi-relational models [28], [44].

Neighbourhood integration. In contrast to post hoc combinations, social networks can be aggregated at an earlier stage to establish a union neighbourhood to perform traditional CF models [16], [29].

In general, post hoc combination approaches combines the outputs of any two techniques using a weighting function such as Arithmetic Mean [25] and Harmonic Mean [23] to aggregate the results. Unified models are often applied by extending the model-based approaches. For example, Jamali and Ester [5] propose an enhanced random walks model to integrate the user-user trust relations with user-item ratings. Tan et al. [27] introduce hypergraphs to handle high-order relationships between users, items and tags. Neighbourhood interaction can be undertaken in a simple way, such as by mixing different neighbourhoods into a union neighbourhood [29]. There are also advanced approaches that involve multi-relational social networks to generate overall neighbourhoods for users [16], [30], but basically they merge user relations into a union network using linear or nonlinear weighting methods. These approaches can be seen as union graph-based approaches that ignore structural information. These studies are closely related to our study but one of the key concerns of them is lacking inter-network comparisons between different social networks. In addition, tuning the weight of every social network may result in high cost model configuration.

\section{SOCIAL NETWORK PROPAGATION}

Trust transitivity in social networks has been widely accepted in existing social recommendation models [2], [3], [13]. We assume that trust transitivity exists not only in explicit social relationships but also in more general user-to-user correlations, e.g., the rating seminaries and more implicit correlations. It is well known that the rating similarity between two users is computed from their ratings to same items, so the result will be inaccurate or possibly non-existent if the number of commonly-shared items is very small. Instead of directly comparing two users' ratings, however, we can infer their "indirect" similarity via a third user if both of them are found sharing similar preferences with the third user. Based on this assumption, this section proposes a random walk-based propagation model for single-relational social networks constructed from explicit social relationships or implicit correlations.

Formally, a social network between all users in a recommender system is denoted by a simple graph, as in the following definition.

Definition 1 (Single social network). In a recommender system, a social network of users is denoted as a graph $G=\{U, E\}$, where $U$ is the entire user set treated as the graph vertices and $E \subseteq U \times U$ is a set of edges where a directed pair of users $\left[u_{1}, u_{2}\right] \in E$ indicates a social connection from user $u_{1}$ to user $u_{2}$.

Social networks may have different forms, such as directed or undirected, weighted or binary relationships. To be consistent, an undirected relationship can be decomposed to two one-way directed relationships. Also, a binary social network can be treated as a special weighted network in which every edge has equal weighting. For a normalized and weighted network, the edges are natively associated with a weighting function $w: E \rightarrow[0,1]$. For a binary network, however, a weighting function is assumed to be $w: E \rightarrow 1$ which gives a constant weight to every visible edge. In this paper, we use $\left[u_{i}, u_{j}\right]$ to denote a directed path from vertex $u_{i}$ to vertex $u_{j}$. We then denote a weighting adjacency matrix $\mathbf{W}$ for a social network $G=\{U, E\}$ with $\mathbf{W}_{i j}=w\left(\left[u_{i}, u_{j}\right]\right)$ if the directed path $\left[u_{i}, u_{j}\right]$ exists and 0 otherwise. 


\section{A. Single Random Walk}

To infer the missing edges in a social network, we introduce a "walk and select" manner following the work of Jamali and Ester [5] to perform random walks on the vertices, as elaborated below.

Given a source user $u_{\text {sou }}$ and a target user $u_{\text {tar }}$, for whom $\left[u_{\text {sou }}, u_{\text {tar }}\right] \notin E$, i.e., there is no edge from $u_{\text {sou }}$ to $u_{\text {tar }}$ in the original social network, a single random walk is started from the source user to reach the target user to infer their indirect relationship, which is denoted as $\tilde{w}\left(\left[u_{\mathrm{sou}}, u_{\mathrm{tar}}\right]\right)$. Let us assume that a runner randomly moves from one node to a neighbour node at each step, and at a time $t$ it has moved to a certain user $u_{i}$. At this time, the runner can choose to keep moving to another node or terminate this walk. This process relates to the walking part of our model. If the runner decides to terminate the walk, the value of $w\left(\left[u_{i}, u_{\mathrm{tar}}\right]\right)$ is returned - a process called the selection part. To summarize, the walking action performs the search of similar users in the network while the selection action polls the suggestions of the reached user's opinion.

In summary, the two options at time $t$ when the runner is located at user $u_{i}$ are as follows.

Option 1. With probability $\phi_{t}$, the walking is terminated and $w\left(\left[u_{i}, u_{\mathrm{tar}}\right]\right)$ is returned as the result. If $u_{i}$ is neither connected to $u_{\text {tar }}$, zero is returned.

Option 2. With probability $1-\phi_{t}$, the walking continues and another node connected by $u_{i}$ will be randomly reached at the next step.

Here, the notation $\phi_{t}$ is the termination probability with regard to walking time/step $t$. In the walking process, the transition probability of moving from a current user $u_{i}$ to another user $u_{j}$ is:

$$
p\left(u_{j} \mid u_{i}\right)=\frac{W_{i j}}{d\left(u_{i}\right)},
$$

where $d\left(u_{i}\right)$ denotes the vertex degree of user $u_{i}$, as in:

$$
d\left(u_{i}\right)=\sum_{j=1}^{|U|} W_{i j} .
$$

A diagonal matrix $\mathbf{D}$ can be constructed with $\mathbf{D}_{i i}=d\left(u_{i}\right)$ on every diagonal. We define a row vector $\mathbf{p}^{(t)}$ presenting the visiting probability distribution over all users at a certain time $t$, i.e., the $i$-th element $p_{i}^{(t)}$ denotes the probability of that the $i$-th user $u_{i}$ is being visited at this time. With these settings, the transition matrix of this random walk is:

$$
\mathbf{T}=\mathbf{D}^{-1} \mathbf{W} \text {. }
$$

If the runner keeps moving at the next time $t+1$, the distribution vector will be updated once as follows.

$$
\mathbf{p}^{(t+1)}=\mathbf{p}^{(t)} \times \mathbf{T}
$$

So far, a single random walk is completed and it returns a prediction of the indirect relationship from the source user to the target user. For more precise predictions, we can issue multiple random walks from the source user and then aggregate all returned values as the final prediction, as detailed in the following section.

\section{B. Performing More Random Walks}

Multiple random walks start from the source user to seek more suggestions for the indirect relationship to the target user. We define a new variable $s$ as the total walking length of a single walk, for which we can obtain a distribution vector $\mathbf{p}^{(s)}$ by updating (4) recursively. We also denote a column vector $W_{\text {tar }}$ as the corresponding column in the weighting matrix $\mathbf{W}$ for the target user $u_{\mathrm{tar}}$. Clearly, $W_{\text {:tar }}$ represents the weightings of the in-linked edges of the target user. The expectation of the returned value for a single random walk terminated at time $s$ should be as follows.

$$
\tilde{w}\left(\left[u_{\mathrm{sou}}, u_{\mathrm{tar}}\right]\right) \mid s=\mathbf{p}^{(s)} W_{\text {:tar }}
$$

Aggregating all random walks that start from the source user, the global expectation of the returned values will be:

$$
\begin{aligned}
\tilde{w}\left(\left[u_{\mathrm{sou}}, u_{\mathrm{tar}}\right]\right) & =p(s=1) \mathbf{p}^{(1)} W_{\mathrm{tar}}+p(s=2) \mathbf{p}^{(2)} W_{\mathrm{tar}} \\
& +p(s=3) \mathbf{p}^{(3)} W_{\mathrm{tar}}+\ldots
\end{aligned}
$$

The starting distribution would be $\mathbf{q}=\mathbf{p}^{(0)}$. As all random walks start from the particular source user $u_{\text {sou }}, \mathbf{q}$ has only one positive element $q\left(u_{\text {sou }}\right)=1$ and all others are zeros. Combining (4) and (6), we have the following simplification.

$$
\begin{aligned}
\tilde{w}\left(\left[u_{\mathrm{sou}}, u_{\mathrm{tar}}\right]\right) & =\phi_{1} \mathbf{q} \mathbf{T} W_{: \mathrm{tar}}+\left(1-\phi_{1}\right) \phi_{2} \mathbf{q} \mathbf{T}^{2} W_{\mathrm{tar}} \\
& +\left(1-\phi_{1}\right)\left(1-\phi_{2}\right) \phi_{3} \mathbf{q} \mathbf{T}^{3} W_{: \mathrm{tar}}+\ldots \\
& =\sum_{t=1}^{\infty} \phi_{t} \prod_{i=1}^{t-1}\left(1-\phi_{i}\right) \mathbf{q} \mathbf{T}^{t} W_{\mathrm{tar}} \\
& \square \sum_{t=1}^{\infty} \psi(t) \mathbf{q} \mathbf{T}^{t} W_{\mathrm{tar}}
\end{aligned}
$$

In the above computation, a new term $\psi(t)$ is introduced, which denotes the probability of a single random walk terminated at time $t$, that is:

$$
\psi(t)=p(s=t \mid \phi)=\phi_{t} \prod_{i=1}^{t-1}\left(1-\phi_{i}\right) .
$$

Equation (7) predicts the indirect connections between two users in a social network. From the perspective of the whole network, we obtain an inferred weighting matrix $\tilde{\mathbf{W}}$, where $\tilde{W}_{i j}$ is the predicted weighting of the relationship between each pair of users. This matrix can be calculated by matrix calculation as follows.

$$
\tilde{\mathbf{W}}=\sum_{t=1}^{\infty} \psi(t) \mathbf{T}^{t} \mathbf{W}
$$

We can prevent walks that are too long term by adjusting the 
termination parameter $\phi$. Based on the idea of "six degrees of separation" [5], [31], most users will be reachable with a walk that is at most six steps in length. Hence, if a walk has reached six steps, we force it to terminate, i.e., let $\phi_{6}=1$. Thus, (9) can be replaced by the following approximation.

$$
\tilde{\mathbf{W}} \approx \sum_{t=1}^{6} \psi(t) \mathbf{T}^{t} \mathbf{W}
$$

It is appropriate to assume the termination probability will become higher when random walks go to deeper levels, that is, parameter $\phi$ increases with time $t$. Simply, we let $\phi_{t}$ increase from 0.5 to 1 in the first six steps, as indicated in Table I, where the distribution of walking length $\psi=p\left(s=t \mid \phi_{t}\right)$ is also computed. We find that most $(80 \%)$ random walks will stop at the first two steps. Based on the Pareto Principle (also known as the 80-20 rule), a fast and approximate solution of (10) is obtained based only on the first two steps, as follows:

$$
\tilde{\mathbf{W}} \approx 0.5 \mathbf{T W}+0.3 \mathbf{T}^{2} \mathbf{W}
$$

TABLE I. AN EXAMPLE SETTING OF THE TERMINATION PARAMETER

\begin{tabular}{ccccccc}
\hline \hline$t$ & 1 & 2 & 3 & 4 & 5 & 6 \\
\hline$\phi_{t}$ & 0.5 & 0.6 & 0.7 & 0.8 & 0.9 & 1 \\
$\psi_{t}=p\left(s=t \mid \phi_{t}\right)$ & 0.5 & 0.3 & 0.14 & 0.048 & 0.011 & 0.001 \\
$p\left(s \leq t \mid \phi_{t}\right)$ & 0.5 & 0.8 & 0.94 & 0.988 & 0.999 & 1 \\
\hline \hline
\end{tabular}

Note that we only need to predict the indirect relationships, so the final weighting matrix after propagation will be:

$$
\hat{\mathbf{W}}=\mathbf{W}+(\mathbf{J}-\mathbf{H}) \circ \tilde{\mathbf{W}},
$$

where $\circ$ is entry-wise production, $\mathbf{J}$ is a matrix with all elements to be 1 with appropriate size and $\mathbf{H}$ is the adjacent matrix of the graph with $H_{i j}=1$ if there is an edge from the $i$-th user $u_{i}$ to the $j$-th user $u_{j}$ and $H_{i j}=0$ otherwise.

TABLE II

RESULT COMPARISON OF THE FULL AND ALTERNATIVE PROPAGATORS

\begin{tabular}{lcc}
\hline \hline \multicolumn{1}{c}{ User relationships } & Similarity & Friendship \\
& $22.4 \%$ & $0.6 \%$ \\
\hline Density after propagation (full) & $79.2 \%$ & $77 \%$ \\
Density after propagation (alternative) & $79.1 \%$ & $39 \%$ \\
Mean deviation of both-inferred edges & 0.047 & 0.006 \\
Mean value of omitted edges & 0.006 & 0.001 \\
\hline \hline
\end{tabular}

We conduct preliminary experiments to evaluate the precision of the alternative calculation of (11) on a real-world dataset of Last.fm [32]. Two relational user networks are collected from the dataset. One is the explicit friendship network of users. The other is a preference similarity network derived from users' listening records. Each network is propagated using the full propagation (10) and the alternative propagation (11), respectively. The experimental results are compared in Table II. First, for the similarity network that has denser original relationships, the density improvements of the two propagators are very close $(79.2 \%$ vs $79.1 \%)$ and the mean deviation of the inferred relationships can be ignored (0.047). The average strength of the omitted edges of the alternative propagator, i.e., those inferred by the full propagator but ignored by the alternative propagator, is only 0.006. The two propagators vary in network density improvement (77\% vs. $39 \%$ ) for the friendship network, but the mean deviation and the omitted values are both trivial (0.006 and 0.001 , respectively). These comparisons demonstrate that (11) is a good alternative to (10) for simplifying the calculation.

To summarize this section, the proposed social network propagation will be applied to every collected single network of users as a pre-processing step to enrich the input data of our multi-relational social network-based recommender system. For convenience of expression, we still use the original notations such as $\mathbf{W}$ instead of $\hat{\mathbf{W}}$ in the remainder of this paper, unless otherwise specified.

\section{Multi-Relational SOCIAL NetwORKS}

This section discusses how to represent different types of user-to-user relationships using a multigraph model.

\section{A. Multigraph Generation}

Formally, let graphs $G_{1}=\left\{U, E_{1}, w_{1}\right\}, G_{2}=\left\{U, E_{2}, w_{2}\right\}$, $\ldots, G_{Z}=\left\{U, E_{Z}, w_{Z}\right\}$ denote the collected and propagated $\mathrm{Z}$ types of social networks on a common user set $U$, which is the population of users in a recommender system, and $E_{1}, E_{2}, \ldots, E_{Z} \subseteq U \times U$ are respectively the edge sets of each graph. A graph $G_{k}$ is associated with a weighting function $w_{k}$ and correspondingly a weighting matrix $\mathbf{W}_{k}$ as denoted.

A simple way to handle multiple social networks is to aggregate different user relationships to build a union graph:

Definition 2 (Union Graph). A union graph is an ordinary simple graph $G^{\prime}=\left\{U, E^{\prime}, w^{\prime}\right\}$ on the vertex set $U$, in which the edges are given by the union set of all single edge sets, i.e., $E^{\prime}=\bigcup_{k=1}^{Z} E_{k}$. Correspondingly, a union weighting function $w^{\prime}: E^{\prime} \rightarrow[0,1]$ is associated to aggregate the available edge weightings of single graphs:

$$
w^{\prime}(e)=g\left(w_{1}(e), w_{2}(e), \ldots, w_{Z}(e)\right), \forall e \in E^{\prime},
$$

where $g$ is an aggregating function such as linear averaging in the study by Jacob et al. [30].

In contrast, a multigraph that retains the original structures of all single graphs is defined as follows [17] .

Definition 3 (Multigraph). A multigraph $G=\{U, E\}$ is a special graph on $U$, where the edge set $E=\underset{k=1}{Z} E_{k}$ is given by the multiset of the edge sets of single graphs $G_{1}$ to $G_{2}$.

As shown in the above definition, a multigraph can be seen as overlapping all single graphs rather than merging them into one simple graph. In the following, we use the subscript symbols $i$ and $j$ to index vertices (users) and $k$ and $l$ to index single graphs.

\section{B. Inter-Network Comparison}

Inter-network comparisons have attracted much study to compare the structural information between different networks. We introduce the Average Similarity of Neighbours (ASN) 
[33], [34] as a measurement of the structural similarity of two networks. This metric was not originally proposed for weighted graphs, thus we modify it to the following form:

$$
\operatorname{ASN}(A, B)=\frac{\sum_{i} \operatorname{DEG}_{A B}(i)}{\sum_{i} \operatorname{DEG}_{A}(i)+\mathrm{DEG}_{B}(i)},
$$

where $\mathrm{DEG}_{A}(i)$ and $\mathrm{DEG}_{B}(i)$ are respectively the out degrees of the $i$-th node in graph $\mathrm{A}$ and graph $\mathrm{B}$ and $\mathrm{DEG}_{A B}(i)$ denotes the summation of the out degrees of this node to the common neighbours in both graphs. Clearly, we have that $\mathrm{DEG}_{A B}(i) \leq \mathrm{DEG}_{A}(i)+\mathrm{DEG}_{B}(i)$ and the equation holds only if the node has the exactly same out-linked neighbours in both graphs.

For recommender systems, we put emphasis on the structural "diversity" rather than the "similarity" measurement of different user networks, given as follows.

$$
\delta(A, B)=1-\operatorname{ASN}(A, B)
$$

The proposed inter-network diversity measurement can be used to pre-screen the various input user relationships. For example, we adopt only one if two networks have very small diversity for the following two reasons. First, the two networks have very similar structure such that it is no need to combine them for the data sparsity problem. Second, incorporating duplicate networks is equivalent to reusing a same information resource, which is unfair for other input resources.
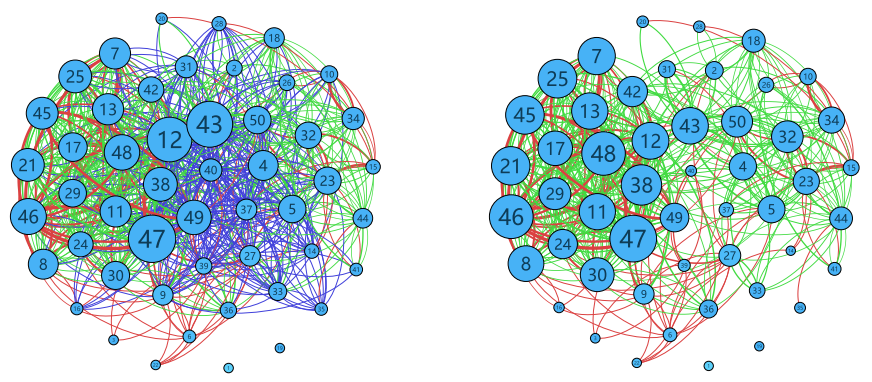

(a) all three types of relationships (b) friendship + similarity

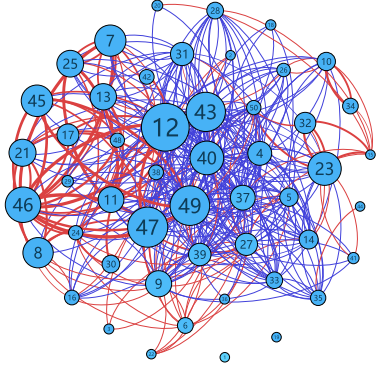

(c) friendship + tagging

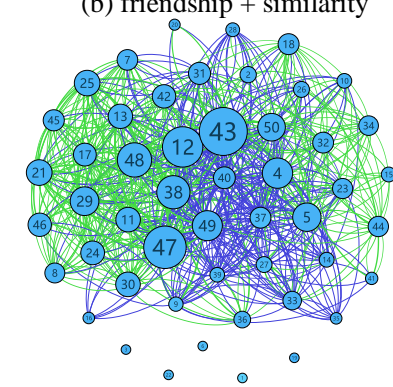

(b) similarity + tagging

Fig.2 Inter-network comparisons with Last.fm dataset (red: friendship, blue: preference similarity, green: co-tagging relationships)

Figure 2 illustrates the network-to-network comparisons in an example data set from Last.fm. We import the complex relationships of the first 50 users in the Last.fm dataset mentioned in the last section. Three relational networks between users are initialized from different resources including the explicit friendships, the preference similarities, and the co-tagging relationships. The overall multigraph structure is presented in Fig.2.(a) by overlapping all three kinds of relationships. We compare each pair from the three single networks to measure their structural diversity in the subfigures Fig.2.(b), Fig.2.(c) and Fig.2.(d), respectively. Fig.2.(b) shows that the friendship and the similarity networks share a small part of the edges. Figure 2.(c) presents the friendship network and tagging network and illustrates that these two networks are also well distinguished. Figure 2.(d) indicates that similarity and tagging networks have more common edges than is shown in the former two figures. We calculate the inter-network diversity measurement of (15) for each pair of the three networks and obtain the following results.

$$
\left\{\begin{array}{l}
\delta \text { (friendship, similarity })=0.82 \\
\delta \text { (friendship, tagging })=0.83 \\
\delta(\text { similarity, tagging })=0.42
\end{array}\right.
$$

The result supports our intuitive observations that the friendship and similarity networks differ in structure, and so do the friendship and tagging networks, while the diversity between the similarity and tagging networks is less, i.e., they are more similar in structure. We should note that the proposed inter-network diversity measurement only evaluates the difference in network structure rather than the quantitative weighting of edges. In other words, "high diversity" here indicates that two networks have complementary structures, i.e., they share a small part of the common edges, but it does not mean that the strength of their common edges are inconsistent. Admittedly, it is helpful to alleviate the data sparsity problem when utilizing more complementary social networks.

\section{MultigRAPH RANKING AND ReCOMMENDATIONS}

A key task of CF is to identify the closed neighbour users who are thought to have similar preferences to the active user. Various user relationships, such as the rating-derived similarity or trust [7], [35], explicit social connections [36] and implicit correlations [11] have been imported as clues to evaluate the closeness between users. Unlike most existing studies, which only apply to a single type of social relationships, this section proposes a multigraph ranking model that can identify the nearest neighbours from multiple social networks.

To illustrate the need for multigraph and its advantage, we start this section with a two-moon ranking problem shown in Fig.3. In this example, the users are connected by three types of relationships, namely, similarity, friendship and tagging correlations, as in the previous example of the Last.fm dataset. User nodes are placed in a geometric figure with regard to the average strength of the three types of relationships with respect to the query user. In addition, those users who are strongly connected with a particular type of relationship are marked with a unique surrounding colour: blue for similarity, red for friendship and green for tagging correlations. In this way, we can locate several small groups of users surrounded by different colours in the figure. Placing all these small groups together, we obtain the whole structure, indicating that two large partitions are generated by adjacent small user groups. We call this problem a two-moon multi-relational social network ranking problem. The goal of this study is to identify the overall nearest neighbours for the particular query user marked in the 
figure. In the following subfigures, Fig.3.(b) to Fig.3.(d), three different ranking ideas are compared. Note that the marker sizes are proportional to the ranking scores of a particular ranker.

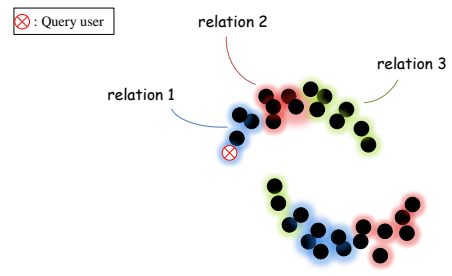

(a) Two-moons partition of multi-relational networks
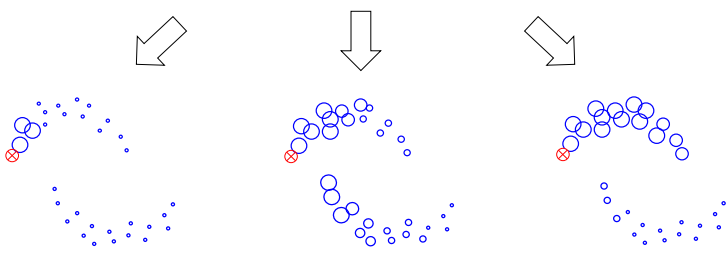

$\begin{array}{lll}\text { (b) Ranking by CF similarity } & \text { (c) Union graph-based ranking } & \text { (d) Multigraph-based ideal ranking }\end{array}$ Fig.3. Ranking in a multi-relational network with a two-moon pattern.

Conventional single network-based approaches find the nearest neighbours who are connected to the query user by a particular type of relationship. For example, Fig.3.(b) presents the expected ranking result of the $\mathrm{CF}$ approach using the preference similarity of users. The $\mathrm{CF}$ method can only find a small number of neighbours because of data sparsity, and is therefore not a successful ranker, as it fails to assess many users. Fig.3.(c) is the ranking result of a union graph-based ranker that uses averaged Euclidian distance. This ranker independently compares the average closeness of the candidate users to the query user, so the connections between the candidates are ignored. As a result, the two-moon pattern cannot be recognized by this kind of ranker. Fig.3.(d) shows the ideal ranking result that we expect to obtain with the proposed multigraph ranking model. This ranker can identify users in the same partition as a result of adjacent small groups with different types of relationships.

A brief preliminary study of simple graph ranking, presented in the next section, is required to grasp the key idea of ranking graph data, and we expand it to the multigraph environment.

\section{A. Simple Graph Ranking}

The graph ranking problem has been studied in considerable depth in recent years and some solutions have been readily adopted in the area of network sampling [37]-[40]. This problem is given by a weighted graph $G=(V, E, w)$, where $V=\left\{v_{1}, \ldots, v_{n}\right\}$ is a set of vertices, $E \subseteq V \times V$ a set of edges, and $w: E \rightarrow[0,1]$ the weighting function, together with an input query vector $\mathbf{y} \in \square^{n}$, in which the $i$-th element $y_{i}$ denotes the initial query score of the node $v_{i}$. The query vector can be seen as a given (input) ranking function $y: V \rightarrow \square$ on the vertex space such that $y\left(v_{i}\right)=y_{i}$. The ranking problem can then be thought of as seeking a new function $f: V \rightarrow \square$ that is smooth and close to the given function $y$ simultaneously. The graph ranking problem is usually formalized to an optimization problem to minimize the following cost function.

$$
\min _{f: V \rightarrow \square} Q(f)=\mathrm{S}(f)+\mu \hat{R}(f ; y)
$$

In the above expression, the first term $\mathrm{S}(f)$ on the right-hand side measures the smoothness of the ranking function $f$; the second term $\hat{R}(f ; y)$ measures the empirical error of $f$ compared to $y$. A trade-off parameter $\mu>0$ is imported to balance the two terms.

The ranking error $\hat{R}(f ; y)$ is usually computed using the $\ell_{2}$ norm variance and is written in matrix-vector form:

$$
\hat{R}(f ; y)=\|f-y\|^{2}=(\mathbf{f}-\mathbf{y})^{T}(\mathbf{f}-\mathbf{y}) .
$$

A good ranking function $f$ will not vary greatly across two vertices that are "closely related". By importing the weighting matrix $\mathbf{W}$ and the degree matrix $\mathbf{D}$ of a simple graph $G$, the regularization framework first proposed in [38] gives the smoothness function as:

$$
S(f)=\mathbf{f}^{T}(\mathbf{I}-\mathbf{A}) \mathbf{f},
$$

where a matrix $\mathbf{A}=\mathbf{D}^{-1 / 2} \mathbf{W D}^{-1 / 2}$ is defined. Requiring the gradient of $Q(\mathbf{f})$ to vanish gives us the following result:

$$
\left.\frac{\partial Q}{\partial \mathbf{f}}\right|_{\mathbf{f}=\mathbf{f}^{*}}=(\mathbf{I}-\mathbf{A}) \mathbf{f}^{*}+\mu\left(\mathbf{f}^{*}-\mathbf{y}\right)=0
$$

In defining a decimal parameter $\alpha=1 /(\mu+1) \in(0,1)$, the optimized ranking result $\mathbf{f}^{*}$ is obtained by solving (20).

$$
\begin{aligned}
\mathbf{f}^{*} & =(1-\alpha)(\mathbf{I}-\alpha \mathbf{A})^{-1} \mathbf{y} \\
& \propto(\mathbf{I}-\alpha \mathbf{A})^{-1} \mathbf{y}
\end{aligned}
$$

The positive constant $1-\alpha$ can be omitted as it does not affect the ranking order.

\section{B. Multigraph Ranking Problem}

We have the same goal for multigraph ranking as for the single graph ranking problem: to seek a good ranking function $f: V \rightarrow \square$ that is smooth and simultaneously close to a given query $\mathbf{y}$. The problem can also be formalized to minimize a cost function in the form of (17). It is clear that the second term $\hat{R}(f ; y)$ is not changed in the multigraph environment, so we only have to elaborate a new smoothness function $\mathrm{S}(f)$ for the multigraph ranking problem.

In a multigraph constructed from several single graphs, the overall relationship of a pair of vertices is represented by a set of edges with regard to different types of relationships. We still use the notations defined in Section III, which supposes that a multigraph $G$ has been established from $Z$ social networks. The relationship between two users can be represented by a column vector containing the edge weighting in each single network, as follows. 


$$
\left[u_{i}, u_{j}\right] \leftarrow\left(\begin{array}{c}
w_{i j}^{1} \\
w_{i j}^{2} \\
\vdots \\
w_{i j}^{Z}
\end{array}\right)
$$

Here we use $w_{i j}^{k} \in[0,1]$ as a simplified expression of $w_{k}\left(\left[u_{i}, u_{j}\right]\right)$, i.e., the closeness of the two users in the k-th graph. Note that $w_{i j}^{k}=0$ if there is no connection between $u_{i}$ and $u_{j}$ in the $k$-th single graph.

An edge function is a function that can map a directed pair of vertices to a real value [30]. We import the edge function to multigraphs, which gives an initial estimation of the strength of the overall relationships between two vertices. To utilize both intra-network relationships and inter-network diversities, we define a virtual edge function $\pi: U \times U \rightarrow \square$ for the user vertices in a multigraph as follows:

$$
\pi\left(\left[u_{i}, u_{j}\right] ; \Delta\right)=\frac{1}{2} \sum_{k, l}^{Z} w_{i j}^{k} w_{i j}^{l} \Delta_{k l},
$$

where $\Delta_{k l}$ is the inter-network diversity between the $k$-th and $l$-th graphs. Comparing the structural diversity of each pair of single graphs, we obtain a $Z \times Z$ diversity matrix $\Delta$ :

$$
\Delta_{k l}= \begin{cases}\delta\left(G_{k}, G_{l}\right), & k \neq l \\ 1, & k=l\end{cases}
$$

This setting considers the unique environment in recommender systems: we assume the relationship of two users should be emphasized if they are connected by many and diverse relationships. We define a matrix $\boldsymbol{\Pi}$ with $\Pi_{i j}=\pi\left(\left[v_{i}, v_{j}\right]\right)$ and it is computed by the following matrices.

$$
\boldsymbol{\Pi}=\frac{1}{2} \sum_{k, l=1}^{Z} \Delta_{k l} \mathbf{W}_{k} \circ \mathbf{W}_{l}
$$

Further, we define the virtual out-degree $\mathrm{d}^{+}\left(u_{i}\right)$ and in-degree $\mathrm{d}^{-}\left(u_{i}\right)$ of a particular user $u_{i}$ as follows.

$$
\begin{aligned}
& \mathrm{d}^{+}\left(u_{i}\right)=\sum_{j \neq i} \pi\left(\left[u_{i}, u_{j}\right]\right) \\
& \mathrm{d}^{\top}\left(u_{i}\right)=\sum_{j \neq i} \pi\left(\left[u_{j}, u_{i}\right]\right)
\end{aligned}
$$

Two diagonal matrices $D_{+}$and $D_{-}$are then denoted with $\left(\mathrm{D}_{+}\right)_{i i}=\mathrm{d}^{+}\left(u_{i}\right)$ and $\left(\mathrm{D}_{-}\right)_{i i}=\mathrm{d}^{-}\left(u_{i}\right)$ on the diagonals.

According to the regularization framework for simple graphs [30], the smoothness of a ranking function consists of the edge derivation crossing every pair of users, which can be defined as follows for a multigraph model.

For an out-linked virtual edge, the edge derivation is:

$$
\frac{\partial f}{\partial\left[u_{i}, u_{j}\right]} \mid=\sqrt{\frac{\pi\left(\left[u_{i}, u_{j}\right]\right)}{\mathrm{d}^{+}\left(u_{i}\right)}} f\left(u_{i}\right)-\sqrt{\frac{\pi\left(\left[u_{i}, u_{j}\right]\right)}{\mathrm{d}^{+}\left(u_{j}\right)}} f\left(u_{j}\right)
$$

For an in-linked virtual edge, the edge derivation is:

$\frac{\partial f}{\partial\left[u_{j}, u_{i}\right]} \mid=\sqrt{\frac{\pi\left(\left[u_{j}, u_{i}\right]\right)}{\mathrm{d}^{-}\left(u_{i}\right)}} f\left(u_{i}\right)-\sqrt{\frac{\pi\left(\left[u_{j}, u_{i}\right]\right)}{\mathrm{d}^{+}\left(u_{j}\right)}} f\left(u_{j}\right)$

Clearly, we have $\left.\frac{\partial f}{\partial\left[u_{i}, u_{j}\right]}\right|_{u_{j}}=-\left.\frac{\partial f}{\partial\left[u_{i}, u_{j}\right]}\right|_{u_{i}}$. Next, the local variation at each vertex in a multigraph is:

$\left\|\nabla_{u_{i}} f\right\|=\sqrt{\frac{1}{2}\left[\sum_{j \neq i}\left(\left.\frac{\partial f}{\partial\left[u_{i}, u_{j}\right]}\right|_{u_{i}}\right)^{2}+\sum_{j \neq i}\left(\left.\frac{\partial f}{\partial\left[u_{j}, u_{i}\right]}\right|_{u_{i}}\right)^{2}\right]}$

The summation of all local variations of all users reflects the overall smoothness of the ranking function [38], which is given by:

$$
\mathrm{S}(f)=\frac{1}{2} \sum_{u \in \mathrm{U}}\left\|\nabla_{u_{i}} f\right\|^{2}
$$

For our multigraph model, the above expression can be simplified in the following steps.

$\mathrm{S}(f)$

$=\frac{1}{2} \sum_{i=1}^{n}\left\|\nabla_{u_{i}} f\right\|^{2}$

$=\frac{1}{4}\left[\sum_{i=1}^{n} \sum_{j=1}^{n}\left(\left.\frac{\partial f}{\partial\left[u_{i}, u_{j}\right]}\right|_{u_{i}}\right)^{2}+\sum_{i=1}^{n} \sum_{j=1}^{n}\left(\left.\frac{\partial f}{\partial\left[u_{j}, u_{i}\right]}\right|_{u_{i}}\right)^{2}\right]$

$=\frac{1}{2} \sum_{i=1}^{n} \sum_{j=1}^{n}\left(\left.\frac{\partial f}{\partial\left[u_{i}, u_{j}\right]}\right|_{u_{i}}\right)^{2}$

$=\frac{1}{2} \sum_{i=1}^{n} \sum_{j=1}^{n}\left(\frac{\pi_{i j}}{\mathrm{~d}_{i}^{+}} f_{i}^{2}+\frac{\pi_{i j}}{\mathrm{~d}_{j}^{-}} f_{j}^{2}-2 \frac{\pi_{i j} f_{i} f_{j}}{\sqrt{\mathrm{d}_{i}^{+} \mathrm{d}_{j}^{-}}}\right)$

$=\frac{1}{2} \sum_{i=1} f_{i}^{2}+\frac{1}{2} \sum_{j=1} f_{j}^{2}-\sum_{i=1}^{n} \sum_{j=1}^{n} \frac{\pi_{i j} f_{i} f_{j}}{\sqrt{\mathrm{d}_{i}^{+} \mathrm{d}_{j}^{\top}}}$

$=\mathbf{f}^{T} \mathbf{f}-\mathbf{f}^{T} \mathrm{D}_{+}^{-1 / 2} \Pi \mathrm{D}_{-}^{-1 / 2} \mathbf{f}$

$=\mathbf{f}^{T}(\mathbf{I}-\mathbf{S}) \mathbf{f}$

In the above calculation, a matrix $\mathbf{S}$ is defined as follows:

$$
\mathbf{S}=\mathrm{D}_{+}^{-1 / 2} \boldsymbol{\Pi} \mathbf{D}_{-}^{-1 / 2} .
$$

The multigraph ranking problem can thus be formalized to the following optimization.

$$
\min _{f: U \rightarrow \square} Q(f)=\mathbf{f}^{T}(\mathbf{I}-\mathbf{S}) \mathbf{f}+\mu(\mathbf{f}-\mathbf{y})^{T}(\mathbf{f}-\mathbf{y})
$$

The new cost function has a similar form to that of single graph ranking, so we can obtain the optimized ranking vector $\mathbf{f}_{m}$ for multigraph ranking following the similar calculations of (21), as follows:

$$
\mathbf{f}_{m}=(\mathbf{I}-\alpha \mathbf{S})^{-1} \mathbf{y}
$$

The same, $\alpha \in(0,1)$ is a model parameter balancing the 
smoothness and consistency of the ranking.

\section{Making Recommendations}

To generate recommendations for an active user $u_{a}$, we hope to identify the closest neighbour users using the proposed multigraph ranking model, which requires a column vector $\mathbf{y} \in \square^{|U|}$ given as the input query vector. The edge function defined in (23) can be natively imported to generate an initial query vector, as follows.

$$
y\left(u_{i}\right)= \begin{cases}\frac{1}{2} Z^{2}, & \text { if } u_{i}=u_{a} \\ \pi\left(\left[u_{a}, u_{i}\right]\right), & \text { if } u_{i} \neq u_{a}\end{cases}
$$

It is easy to find that $\pi \leq \frac{1}{2} Z^{2}$ from (23). With the query vector $\mathbf{y}$, the optimized ranking vector $\mathbf{f}$ will be obtained by solving (35). The top-K users who acquire the highest ranking scores are then selected as the nearest neighbours for the active user, denoted as $\operatorname{Neib}\left(u_{a}\right)$. Next, the standard CF prediction formula is imported to predict the possible rating of the active user $u_{a}$ to an unseen item $i$ [35].

$$
\hat{r}\left(u_{a}, i\right)=\bar{r}_{u_{a}}+\frac{\sum_{u \in \operatorname{Neib}\left(u_{a}\right)} f(u)\left(r_{u, i}-\bar{r}_{u}\right)}{\sum_{u \in u_{a}} f(u)}
$$

Ultimately, several (e.g., 5 or 10) of the unseen items with the highest predictions are selected as final recommendations.

We now summarize the whole process of the proposed recommendation approach. First, we collect various explicit or implicit social relationships that may appear in a recommender system and build multiple single social networks for users. Next, the proposed random walk-based social network propagation model is employed to enrich the original data of each social network. After this, a multigraph is constructed to represent the multi-relational social networks of users. Given a particular active user as the requester for recommendations, the proposed multigraph ranking model is then implemented to identify this user's closest neighbour users and the CF rating predictions are made for unseen items. Ultimately, the items with the highest predictions are selected as a list of recommendations to present to the active user.

\section{EXPERIMENTS}

Empirical experiments are conducted with two real-world datasets to compare the performance of our model with existing social recommender systems.

\section{A. Experiment Setup}

We chose two datasets from Epinions.com and Last.fm for experiments. These are two of only a few publicly available datasets that contain both ratings and social network data, and they have been widely used to evaluate social recommender systems [3], [5], [6], [29]. Epinions.com is a general product review site on which customers can rate and review different domains of products including cars, books, movies, and software. The ratings range from 1 (the worst) to 5 (the best). In addition, there is a "web of trust" network recording user relationships of "who trusts whose opinions" - the system enables every user to build a trust list and add people who share similar opinions. The version ${ }^{1}$ of data firstly crawled by Massa and Avesani [3] is imported in our experiments. The statistical information of this dataset is presented in Table III, from which indicates we can see the ratings are very sparse. As a result of this high rating sparsity (99.99\%), pure CF approaches suffer severely from the cold-start problem[3]. This is common in e-Commerce sites such as Amazon.com [41] and Yelp.com [42]. The need to integrate new information such as trust to enhance recommendation quality is therefore highlighted.

TABLE III.

STATISTICAL INFORMATION ABOUT THE EPINIONS DATASET

\begin{tabular}{cccc}
\hline \hline & Total & per-user & Sparsity \\
\hline \#Users & 49290 & - & - \\
\#Products & 139738 & - & - \\
\#Ratings & 664824 & 13.5 & $99.99 \%$ \\
\#Trust & 487181 & 9.9 & $99.98 \%$ \\
\hline \hline
\end{tabular}

TABLE IV.

STATISTICAL INFORMATION ABOUT THE LAST.FM DATASET

\begin{tabular}{cccc}
\hline \hline & Total & per-user & Sparsity \\
\hline \#Users & 2100 & - & - \\
\#Artists (items) & 18745 & - & - \\
\#Listening count (rating) & 92834 & 44.2 & $99.76 \%$ \\
\#Friendship & 25424 & 12.1 & $99.42 \%$ \\
\hline \hline
\end{tabular}

The other dataset is a music sharing dataset ${ }^{2}$ from Last.fm, provided by Cantador et al. [32]. There is no explicit rating information in this dataset but the "listening count" logs of users are often seen as implicit "ratings" of music tracks [27]. In addition, an explicit online friendship network of users is provided in the dataset. Last.fm users are freely able to assign tags to describe music tracks or artists, and it has been reported that a tag-derived social network of users can be extracted for recommendations [43], [10]. Following the settings of Zhen et al. [43], we initialize a co-tagging network between users as another social network besides the friendship network. Statistics about this dataset are summarized in Table IV, which indicates that this dataset has very dense rating data. For convenience, we transform the listening counts to ratings in the following way. For every user, the top one fifth of artists most listened to are assumed to have a 5-star rating (most loved), the second fifth are given a 4-star rating, and so on. The ratings generated for the Last.fm dataset range thus from 1 to 5 , as in the Epinions dataset.

Table V shows the two types of user relationships collected in the Epinions dataset and the three types of user relationships collected in the Last.fm dataset.

TABLE V. DIFFERENT USER-USER RELATIONSHIPS IN TWO DATASETS

\begin{tabular}{ccc}
\hline \hline User relationships & Epinions & Last.fm \\
\hline Rating Similarity & $\checkmark$ & $\checkmark$ \\
Social Trust & $\checkmark$ & $\checkmark$ \\
Social Friendship & & $\checkmark$ \\
Tagging correlation & & 3 \\
\hline No. of networks (Z) & 2 & \\
\hline \hline
\end{tabular}

\footnotetext{
${ }^{1}$ http://www.trustlet.org/wiki/Downloaded_Epinions_dataset

${ }^{2} \mathrm{http}: / / g r o u p l e n s . o r g / d a t a s e t s /$ hetrec-2011/
} 
Our proposed multigraph ranking-based recommendation approach is abbreviated to MGrank, and we compare it in the experiment with the following single or hybrid approaches. Each original social network is first used to implement a user-based CF approach by relying on this single type of user relationships. We also implement an enriched version of each social network using our proposed propagation method in Section III. By comparing the original and propagated social network-based approaches, we can evaluate whether the proposed propagation model can improve recommendation performance. The following single approaches are labelled.

- sCF and sCFPro: single approaches using the original and propagated Cosine similarity networks for collaborative filtering (for both datasets).

- sTrust and sTrustPro: single approaches using the original and propagated trust network (for the Epinions dataset only).

- sFriend and sFfriendPro: single approaches using the original and propagated friendship network (for the Last.fm dataset only).

- sTag and sTagPro: single approaches using the original and propagated tag-derived user social network (for the Last.fm dataset only).

According to our taxonomy for the three fusion strategies reviewed in Section II, we select a representative hybrid approach from each as a benchmark approach for comparison:

- Post hoc combination: The post hoc combination model of Shambour et al. [13] is selected, in which the harmonic mean is used to aggregate the prediction result of each single approach. This approach is denoted as PostCB.

- Unified model-based: The TrustWalker model proposed by Jamali and Ester [5] is selected. Note that this model can only incorporate a single social network; the tagging information in the Last.fm dataset is not included. The name is simplified as TrustWK.

- Neighbourhood integration-based: The union graph, according can be seen as a neighbourhood integration model. Here, the arithmetic average of different relationships is used to set the weighting of the union edges. This approach is denoted as Union.

The recommendation coverage and RMSE (Root Mean Squared Error) are selected as the two evaluation metrics for our experiments.

Recommendation coverage evaluates the successful rate of recommender systems, which is defined as:

$$
\text { Coverage }=\frac{\left|\left\{(u, i) \mid R_{u, i}, P_{u, i}\right\}\right|}{\left|\left\{(u, i) \mid R_{u, i}\right\}\right|},
$$

where $R_{u, i}$ and $P_{u, i}$ are Booleans indicating whether user $u$ ratings item $i$ and this rating is successfully predicted, respectively.

RMSE evaluates the rate of prediction error, defined as:

$$
\mathrm{RMSE}=\sqrt{\frac{\sum_{(u, i) \mid R_{u, i}, P_{u, i}}\left(r_{u, i}-p_{u, i}\right)^{2}}{\left|\left\{(u, i) \mid R_{u, i}, P_{u, i}\right\}\right|}},
$$

where $r_{u, i}$ is the actual rating of user $u$ to an item $i$, and $p_{u, i}$ is the possible rating predicted by a recommendation approach.

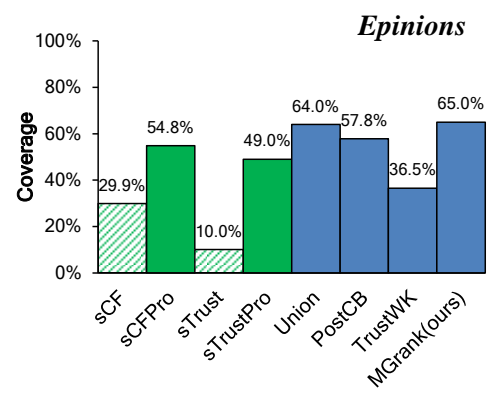

Fig.4. Comparison of recommendation coverage (Epinions dataset)

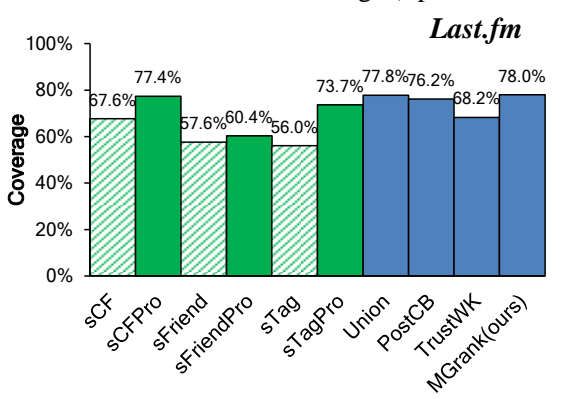

Fig.5. Comparison of recommendation coverage (Last.fm dataset)

\section{B. Performance Comparison}

We collect the recommendation coverage measurements of all approaches in Fig.4 (Epinions dataset) and Fig.5 (Last.fm dataset). In overall, the two figures demonstrate that our approach achieves the highest coverage rate. Focusing on the comparison of the recommendation coverage of single approaches, we find significant improvements after the use of social network propagation, especially when the original social network is sparse. This finding demonstrates the success of the unique random walk-based social network propagation model in alleviating the data sparsity problem. In the Epinions dataset, the original single approaches are found to suffer heavily from the sparsity problem; for example, the sCF completes only $30 \%$ of predictions while sTrust performs even worse (10\%). By implementing network propagation, the coverage rates of the enhanced single approaches are increased to 55\% (sCFPro) and $49 \%$ (sTrustPro), respectively. Similar improvements are also observed in the experiments on the Last.fm dataset, e.g., the coverage of $\mathrm{CF}$ is increased from $68 \%$ to $76 \%$ after social network propagation. In addition, Fig. 4 and Fig. 5 show that hybrid approaches incorporating multiple social networks performs higher coverage rates than single approaches. This demonstrates that the incorporation of different types of user relationships helps to alleviate the sparsity problem of single resource-based recommendation models. In the Epinions dataset, the highest recommendation coverage scores is achieved by MGrank (65\%), followed by Union (64\%) and PostCB (58\%). These hybrid approaches outperforms single approaches significantly such as $\mathrm{SCF}$ and sTrust. In the Last.fm dataset with more and denser user relationships, hybrid approaches achieve high coverage rates of $76 \%$ to $78 \%$, and the MGrank approach maintains the best performance. 


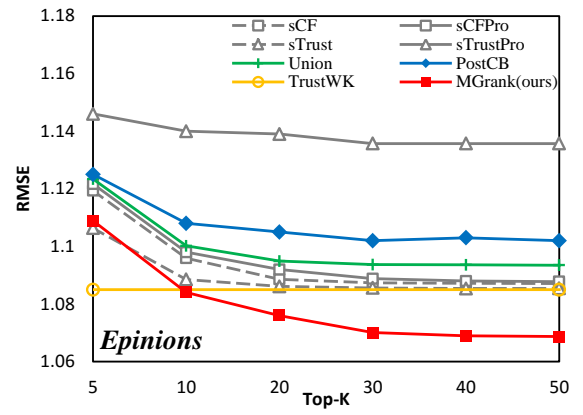

Fig.6. RMSE measurement on the Epinions dataset

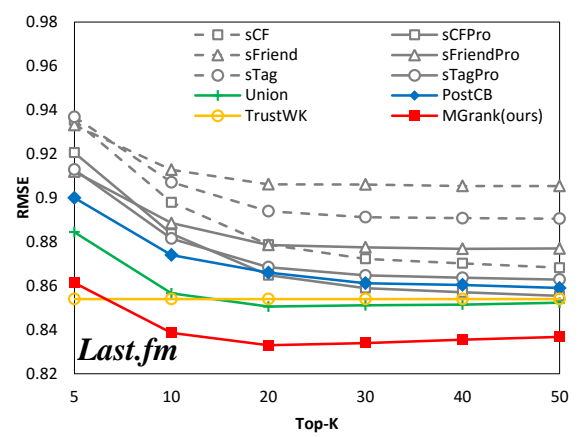

Fig.7. RMSE measurement on the Last.fm dataset

Figures 6 and Fig. 7 present the RMSE measurements along with different settings of neighbourhood size, top-K. Note that TrustWK is not a K-nearest-neighbour model, so its RMSE scores do not vary with $\mathrm{K}$ in the figures. Figure 6 shows that sCFPro and sTrustPro perform even higher errors than $\mathrm{sCF}$ and sTrust that without social network propagations on the Epinions dataset. Even the hybrid approaches Union, PostCB and TrustWK that incorporate multiple social networks are hard to reduce prediction errors. This finding agrees with the conclusions of previous studies that simply averaging different social networks is difficult to maintain recommendation accuracy when the original data are too sparse [3]. In contrast, by introducing multigraph and multigraph ranking, the MGrank approach is able to reduce prediction error rate significantly.

The Last.fm dataset has relatively richer data, and we find that social network propagation is effective in reducing prediction errors, referring to the performances of sCF and sCFPro for an example. In addition, most hybrid approaches achieve lower errors than single approaches. This indicates that incorporating different resources is successful in this dataset. MGrank still achieves significant improvement compared to all other approaches. It is also noteworthy to analyse the performance of the three hybrid approaches, which represent different information fusion strategies of conventional social recommendations. It is found that the post hoc combination approach (PostCB) is able to improve recommendation coverage but cannot guarantee precision. The union graph-based approach (Union) suffers the same problem. Essentially, both of PostCB and Union are hybrid approaches that simple average different information resources. The model-based approach TrustWK generally outperforms PostCB and Union in terms of accuracy, but the improvement in recommendation coverage is limited, especially in sparse environments. Comparing to the traditional hybrid approaches, the proposed multigraph ranking model effectively improves recommendation performance in terms of both accuracy and success rate, especially in sparse environments.

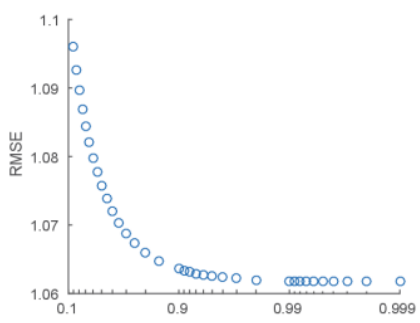

(a) Epinions dataset

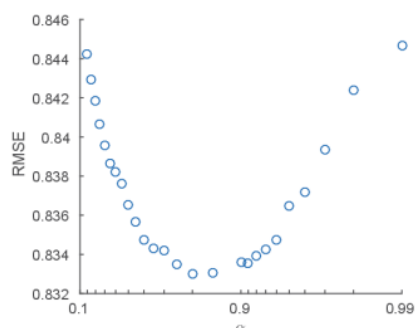

(b) Last.fm dataset
Fig.8 Performance variation with different parameter settings

\section{Parameter Setting}

For the proposed multigraph ranking model, a trade-off parameter $\alpha$ needs to be tuned. We measure the RMSE with increasing values of $\alpha$ on both datasets. Figure 8.(a) shows a gradual decreasing trend of RMSE on the Epinions dataset. We can see the error maintains the lowest level when $\alpha$ reaches 0.99 , equally $\mu=0.01$ in the cost function (34). This indicates that the structural cost (the first part of the equation) should be afforded the most consideration, while the experience cost (the second part) should be weighted less.

On the Last.fm dataset, lowest RMSE is reached at $\alpha=0.8$, followed by a sharp increase when $\alpha$ goes higher, as shown in Fig.8.(b). Optimization is thus obtained at $\mu=0.25$. This indicates the best balance of the cost functions (34) for the Last.fm dataset.

The above discussions provide guidelines for choosing an appropriate trade-off parameter. As a result of the high level of data sparsity, such as in Epinions, a less accurate query vector $\mathbf{y}$ is initialized so that less consideration is given to the experience cost; equivalently, parameter $\alpha$ should be tuned higher in this circumstance. Conversely, if the data is as dense as in Last.fm, the initial query vector becomes informative and accurate; the ranking result $\mathbf{f}$ is thus required to maintain consistency with the query vector. Parameter $\alpha$ should therefore be tuned lower if the input data are sufficient.

\section{CONCLUSIONS AND FURTHER STUDY}

Collaborative filtering has been successfully implemented in recommender systems to find potential items for users. As a result of relying only on rating data, however, the conventional CF model suffers from the cold start problem if ratings are too sparse. To overcome this drawback, an increasing number of social recommender systems import user relationships as an alternative or additional resource for recommendations. This paper differs from previous works because it puts forward a method to handle situations in which users are simultaneously connected by multiple social networks, which is increasingly common as a result of the development of online social networking techniques. This paper introduces multigraph and multigraph ranking model to generate recommendations. First, the multigraph is imported to retain the original structural 
information of multi-relational social networks between users. An inter-network diversity measurement is also provided for better evaluating the structural complementarity of different social networks. We then address a multigraph ranking problem in which the intra-network relationships and inter-network diversities are both considered. By solving this problem we can identify the overall closeness of users from a variety of explicit and implicit correlations between users. The multigraph ranking model is believed to be capable to enhance recommendation performance in terms of both success rate and accuracy, according to the results of a series of experiments on two real-world datasets. It is also noteworthy that this paper proposes a unique random walk-based propagation model for a single social network, as a pre-processing step to enrich the original social data. Empirical experiments indicate that the propagation model is able to increase network density significantly.

Negative relationships such as "distrust" between users are not considered yet, which would be a limitation of this study. The combination of positive and negative relationships remains a topic for future research.

\section{ACKNOWLEDGEMENT}

This work is supported by the Australian Research Council (ARC) under discovery grant DP140101366 and China National Natural Science Foundation grant 71271095.

\section{REFERENCES}

[1] Y. Bo, Y. Lei, D. Liu, and J. Liu. "Social collaborative filtering by trust." In Proceedings of the 23rd International Joint Conference on Artificial Intelligence, pp. 2747-2753, 2013.

[2] J. Golbeck, "Combining provenance with trust in social networks for semantic web content filtering," in Provenance and Annotation of Data, Springer, 2006, pp. 101-108.

[3] P. Massa and P. Avesani, "Trust-aware recommender systems," in The 2007 ACM Conference on Recommender systems, 2007, pp. 17-24.

[4] H. Ma, H. Yang, M. R. Lyu, and I. King, "Sorec: Social recommendation using probabilistic matrix factorization," in Proceedings of the 17th ACM Conference on Information and Knowledge Management, 2008, pp. 931-940.

[5] M. Jamali and M. Ester, "Trustwalker: A random walk model for combining trust-based and item-based recommendation," in The 15th ACM SIGKDD International Conference on Knowledge Discovery and Data Mining, 2009, pp. 397-406.

[6] X. Yang, H. Steck, Y. Guo, and Y. Liu, "On top-k recommendation using social networks," in The 6th ACM Conference on Recommender Systems, New York, NY, USA, 2012, pp. 67-74.

[7] J. O'Donovan and B. Smyth, "Trust in recommender systems," in Proceedings of the 10th International Conference on Intelligent User Interfaces, 1040870, 2005, pp. 167-174.

[8] K. Shiratsuchi, S. Yoshii, and M. Furukawa, "Finding unknown interests utilizing the wisdom of crowds in a social bookmark service," in Proceedings of the IEEE/WIC/ACM International Conference on Web Intelligence and Intelligent Agent Technology, 2006, pp. 421-424.

[9] C.-S. Hwang and Y.-P. Chen, "Using trust in collaborative filtering recommendation," in New Trends in Applied Artificial Intelligence, Springer, 2007, pp. 1052-1060.
[10] H. Liang, Y. Xu, Y. Li, and R. Nayak, "Personalized recommender system based on item taxonomy and folksonomy," in The 19th ACM International Conference on Information and Knowledge Management, New York, 2010, pp. 1641-1644.

[11] G. R. Lopes, M. M. Moro, L. K. Wives, and J. P. M. De Oliveira, "Collaboration recommendation on academic social networks," in Advances in Conceptual Modeling-Applications and Challenges, Springer, 2010, pp. 190-199.

[12] W. Yuan, L. Shu, H.-C. Chao, D. Guan, Y.-K. Lee, and S. Lee, "ITARS: Trust-aware recommender system using implicit trust networks," IET Communications, vol. 4, no. 14, pp. 1709-1721, 2010.

[13] Q. Shambour and J. Lu, "A trust-semantic fusion-based recommendation approach for e-business applications," Decision Support Systems, vol. 54, no. 1, pp. 768-780, 2012.

[14] M. Eirinaki, M. D. Louta, and I. Varlamis, "A trust-aware system for personalized user recommendations in social networks," IEEE Transactions on Systems, Man, and Cybernetics: Systems, vol. 44, no. 4, pp. 409-421, Apr. 2014.

[15] I. Katakis, N. Tsapatsoulis, F. Mendez, V. Triga, and C. Djouvas, "Social voting advice applications - definitions, challenges, datasets and evaluation," IEEE Transactions on Cybernetics, vol. 44, no. 7, pp. 1039-1052, 2014

[16] P. Kazienko, K. Musial, and T. Kajdanowicz, "Multidimensional social network in the social recommender system," IEEE Transactions on Systems, Man and Cybernetics, Part A: Systems and Humans, vol. 41, no. 4, pp. 746-759, 2011.

[17] M. Gjoka, C. T. Butts, M. Kurant, and A. Markopoulou, "Multigraph sampling of online social networks," IEEE Journal on Selected Areas in Communications, vol. 29, no. 9, pp. 1893-1905, 2011.

[18] M. Pazzani and D. Billsus, "Content-based recommendation systems," in The Adaptive Web, vol. 4321, P. Brusilovsky, A. Kobsa, and W. Nejdl, Eds. Springer Berlin Heidelberg, 2007, pp. 325-341.

[19] P. Resnick and H. R. Varian, "Recommender systems," Commun. ACM, vol. 40, no. 3, pp. 56-58, 1997.

[20] B. Sarwar, G. Karypis, J. Konstan, and J. Riedl, "Item-based collaborative filtering recommendation algorithms," in Proceedings of the 10th International Conference on World Wide Web, 2001, pp. 285-295.

[21] M. Gori and A. Pucci, "ItemRank: A random-walk based scoring algorithm for recommender engines," in The 20th International Joint Conference on Artifical Intelligence, San Francisco, CA, USA, 2007, pp. 2766-2771.

[22] J. O'Donovan, "Capturing trust in social web applications," in Computing with Social Trust, Springer London, 2009, pp. 213-257.

[23] Q. Shambour, "Hybrid Recommender Systems for Personalized Government-to-Business e-Services," University of Technology, Sydney, 2012.

[24] J. Golbeck and J. Hendler, "Filmtrust: Movie recommendations using trust in web-based social networks," in Proceedings of the IEEE Consumer Communications and Networking Conference, 2006, vol. 96, pp. 282-286.

[25] M. Claypool, A. Gokhale, T. Miranda, P. Murnikov, D. Netes, and M. Sartin, "Combining content-based and collaborative filters in an online newspaper," in Proceedings of ACM SIGIR Workshop on Recommender Systems, 1999, vol. 60.

[26] A. S. Lampropoulos, P. S. Lampropoulou, and G. A. Tsihrintzis, "A cascade-hybrid music recommender system for mobile services based on musical genre classification and personality diagnosis," Multimedia Tools and Applications, vol. 59, no. 1, pp. 241-258, 2012.

[27] S. Tan, J. Bu, C. Chen, B. Xu, C. Wang, and X. He, "Using rich social media information for music recommendation via 
hypergraph model," ACM Transactions on Multimedia Computing, Communications and Applications, vol. 7S, no. 1, pp. 22:1-22:22, 2011.

[28] M. Jiang, P. Cui, F. Wang, Q. Yang, W. Zhu, and S. Yang, "Social recommendation across multiple relational domains," in Proceedings of the 21st ACM International Conference on Information and Knowledge Management, 2012, pp. 1422-1431.

[29] A. Bellogín, I. Cantador, F. Díez, P. Castells, and E. Chavarriaga, "An empirical comparison of social, collaborative filtering, and hybrid recommenders," ACM Transactions on Intelligent Systems and Technology, vol. 4, no. 1, p. 14, 2013.

[30] Y. Jacob, L. Denoyer, and P. Gallinari, "Classification and annotation in social corpora using multiple relations," in Proceedings of the 20th ACM International Conference on Information and Knowledge Management, 2011, pp. 1215-1220.

[31] S. Milgram, "The small world problem," Psychology Today, vol. 2, no. 1, pp. 60-67, 1967.

[32] I. Cantador, P. Brusilovsky, and T. Kuflik, "Second workshop on information heterogeneity and fusion in recommender systems (HetRec2011).," in The 5th ACM Conference on Recommender Systems, Chicago, IL, USA, 2011, pp. 387-388.

[33] V. Marceau, P.-A. Noël, L. Hébert-Dufresne, A. Allard, and L. J. Dubé, "Modeling the dynamical interaction between epidemics on overlay networks," Physical Review E, vol. 84, no. 2, pp. 026-105, 2011.

[34] D. Zhao, L. Li, H. Peng, Q. Luo, and Y. Yang, "Multiple routes transmitted epidemics on multiplex networks," Physics Letters A, vol. 378, no. 10, pp. 770-776, Feb. 2014.

[35] P. Resnick, N. Iacovou, M. Suchak, P. Bergstrom, and J. Riedl, "GroupLens: An open architecture for collaborative filtering of netnews," in The 1994 ACM Conference on Computer Supported Cooperative Work, 1994, pp. 175-186.

[36] P. Massa and P. Avesani, "Trust metrics in recommender systems," in Computing with social trust, Springer, 2009, pp. 259 285.

[37] M. Belkin, I. Matveeva, and P. Niyogi, "Regularization and semi-supervised learning on large graphs," in Learning theory, Springer, 2004, pp. 624-638.

[38] D. Zhou and B. Schölkopf, "A regularization framework for learning from graph data," in Workshop on Statistical Relational Learning at the 21st International Conference on Machine Learning, Canada, 2004.

[39] S. Agarwal, "Ranking on graph data," in The 23rd International Conference on Machine learning, 2006, pp. 25-32.

[40] K. Mao, J. Fan, L. Shou, G. Chen, and M. Kankanhalli, "Song recommendation for social singing community," in Proceedings of the ACM International Conference on Multimedia, 2014, pp. 127-136.

[41] J. McAuley and J. Leskovec, "Hidden factors and hidden topics: Understanding rating dimensions with review text," in The 7th ACM Conference on Recommender Systems, New York, NY, USA, 2013, pp. 165-172.

[42] J. Blomo, M. Ester, and M. Field, "Recommender system challenge 2013," in Proceedings of the 7th ACM Conference on Recommender systems, 2013, pp. 489-490.

[43] Y. Zhen, W.-J. Li, and D.-Y. Yeung, "TagiCoFi: Tag informed collaborative filtering," in Proceedings of the third ACM Conference on Recommender systems, 2009, pp. 69-76.

[44] M. Jiang, P. Cui , N. J. Yuan, X. Xie, \& S. Yang. "Little Is Much: Bridging Cross-Platform Behaviors through Overlapped Crowds." In 30th AAAI Conference on Artificial Intelligence. 2016.

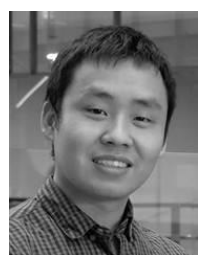

Mingsong Mao is now working at Jiangxi University of Finance and Economics, Nanchang, China. He was a joint $\mathrm{Ph} . \mathrm{D}$ candidate of the University of Technology Sydney, Australia and Huazhong University of Science and Technology (HUST), China. His research interests include recommender systems, social networks, e-business and uncertain information processing.

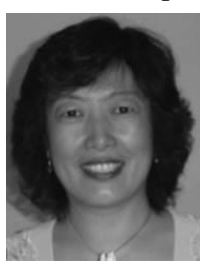

Jie $\mathrm{Lu}$ is a full professor and Associate dean of the Faculty of Engineering and Information Technology at the University of Technology Sydney. Her research interests lie in the area of decision support systems and uncertain information processing. She has published five research books and 270 papers, won five Australian Research Council discovery grants and 10 other grants. She received a University

Research Excellent Medal in 2010. She serves as Editor-In-Chief Knowledge-Based Systems (Elsevier), and International Journal of Computational Intelligence Systems (Atlantis), editor for book series on Intelligent Information Systems and guest editor of special issues for international journals, as well as delivered keynote speeches at international conferences.

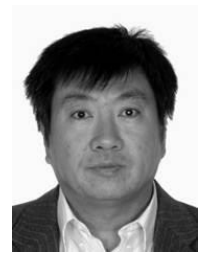

Guangquan Zhang is an associate professor in Faculty of Engineering and Information Technology at the University of Technology Sydney, Australia. $\mathrm{He}$ has a PhD in Applied Mathematics from Curtin University of Technology, Australia. His main research interests lie in the area of multi-objective, bilevel and group decision making, decision support system tools, fuzzy measure, fuzzy optimization and uncertain information processing. He has published four monographs, four reference books and over 200 papers in refereed journals and conference proceedings and book chapters. He has won four Australian Research Council (ARC) discovery grants and many other research grants.

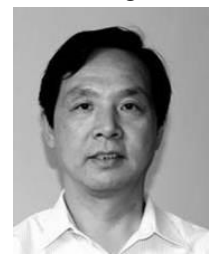

Jinlong Zhang is full professor of the school of management of Huazhong University of Science and Technology, China. His main areas of research interests include modern management theory, information system, managerial innovation and decision-making. He has published over 120 papers in international refereed journals on the above areas. $\mathrm{He}$ is the chief-editor of the Chinese Journal of Management. He is the member of the Advisory Committee of the Institute of Chinese Soft Science, etc. He has actively engaged in management consultant projects for corporations and organizations in China, on strategy management, inventory control and investment appraisals. 